\title{
UN ANÁLISIS DE LA ORIENTACIÓN PROFESIONAL DESDE LA TECNOÉTICA EDUCATIVA
}

\author{
AN ANALYSIS OF THE CAREER GUIDANCE FROM \\ THE EDUCATIONAL TECHNOETHICS
}

\author{
Pilar Alejandra Cortés Pascual*
}

\section{RESUMEN}

La orientación profesional hay que situarla en un contexto sociohistórico concreto que actualmente se caracteriza por la Sociedad de la Información. Desde aquí, nos planteamos que un contenido relativo a la orientación profesional ha ser la comprensión de un análisis ético de las tecnologías y los medios de comunicación, a los que se ha de enfrentar un sujeto en su desarrollo profesional. Esta idea se concretiza en el concepto de tecnoética educativa, y se aborda desde dos parámetros: los valores intrínsecos que incluyen las tecnologías y los medios de comunicación (fin de la tecnoética), y uso de éstos como mediadores de los valores éticos (medio de la tecnoética). Desde ambos sentidos se presenta concisamente la propuesta que se implementa en la actualidad mediante el proyecto "Laboratorio de Observación sobre Tecnoética para Adultos" (LOTA) y sus implicaciones en la orientación profesional.

Palabras claves: tecnologías, medios de comunicación, valores éticos, orientación profesional, tecnoética educativa.

\begin{abstract}
Career Guidance should be set within a specific socio-historical context, which is nowadays characterized by the Society of Information. From this starting point, we think that the understanding of both an ethical analysis of technology as well as of the means of communication, which individuals will have to deal with in their professional development, must be considered as a content linked to career guidance. This idea becomes more definite in the concept of educational technoethics and it is studied from two parameters: the intrinsic values that technology and the mass-media include (the aim of

\footnotetext{
* Profesora Contratada Doctora del área MIDE (Facultad de Educación en la Universidad de Zaragoza), sus líneas de investigación y publicaciones versan en la educación en los valores desde la teoría ecológica de Bronfenbrenner, las tecnologías desde un análisis ético, los valores laborales desde la Orientación Profesional y, la comunicación con los adolescentes y pautas de orientación. Es docente en materias de orientación y también trabajo en temas de innovación docente. alcortes@unizar.es
} 
technoethics) and their use as mediators of ethical values (means of technoethics). Therefore, the proposal that is currently being implemented in the project "Observation Laboratory on Technoethics for Adults" (LOTA) as well as its implications for career guidance are concisely presented from both points of view.

Key words: technology, mass-media, ethical values, career guidance, and educational technoethics.

Para mi madre

\section{Introducción}

La creciente demanda de una mayor profesionalización de los alumnos ha dado lugar a una intensa incorporación de las tecnologías de la información y de la comunicación (TIC) en las aulas. La sociedad de la información implica competencias profesionales generales y en algunos casos específicas en las TIC. Bien es cierto que debido a esta impronta, las tecnologías se están empleando como un recurso para la búsqueda de empleo y formación, sobre todo mediante páginas web y algunos programas on-line e informáticos. Asimismo un objetivo y contenido de la orientación profesional es el de conocer (orientar en saber) y saber utilizar (orientar en saber hacer) los medios tecnológicos y medios de comunicación para la adaptación laboral (Cogoi, Sobrado, Hawthorm, R. y Korte, 2005). En concreto, respecto a las competencias internacionales para los orientadores aprobadas por AIOSP (2003) relativas al career development y placement, ambas categorías están próximas a la orientación profesional, y aunque sugieren el uso de recursos informáticos y en red para dicho ámbito (saber hacer), a nuestro modo de ver también podría incluirse el componente que aquí añadimos del saber ser.

Estas relaciones entre las TIC y la orientación profesional son necesarias, pero se propone la interrelación de un tercer componente: los valores éticos. La razón de estudiar el triángulo construido por la orientación profesional, la educación en valores y tecnología está motivada por acontecimientos sociocontextuales que enmarcan el panorama educativo presente, como son el pensamiento postmoderno y la sociedad del conocimiento y de la información. Es decir, si hablamos del espacio que componen este trío de variables es porque la propia sociedad lo exige y educativamente habremos de dar alguna respuesta. Al respecto, la relación entre orientación profesional, educación en valores y tecnología, a nuestro criterio, se encamina en dos líneas de estudio: la primera sobre la profundización en los valores ocupacionales que exige la sociedad del conocimiento y tecnológica actual (Cortés, 2006), y la segunda acerca de la tecnoética como componente de la orientación profesional (orientar en saber ser). Es decir, la orientación profesional habrá de intervenir, asesorar, aconsejar, programar o dar respuesta a una consulta en tres direcciones: saber sobre TIC, saber utilizar las TIC y saber ser con las TIC. En la gráfica 1 se representa lo expuesto:

\section{Contextualización del trabajo}

En este trabajo se profundiza en la última dirección, es decir, en orientar académica y profesionalmente en los contenidos éticos que conlleva el uso de las tecnologías, es decir, orientar en la tecnoética. Se comienza por la tecnoética educativa, concepto que hemos de- 


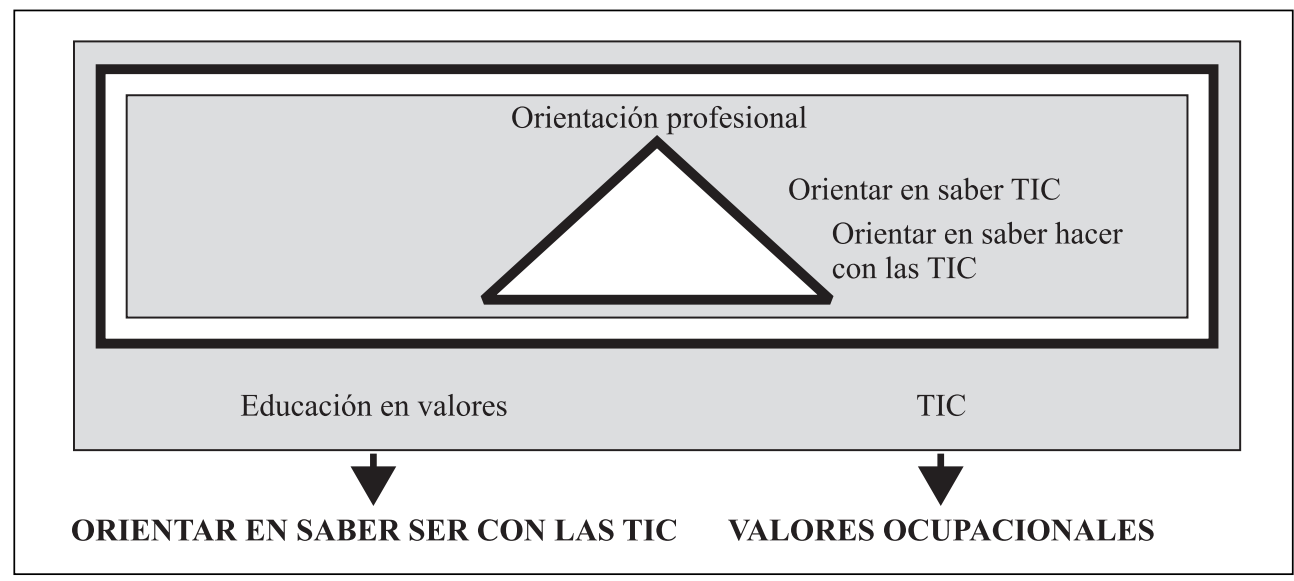

GRÁFICA 1.

Triángulo entre orientación profesional, educación en valores y TIC.

sarrollado en anteriores trabajos (Cortés, 2005a; 2006) y que aquí también lo integraremos con el campo de la orientación profesional. Al comenzar a precisar esta idea se aprecia que parte de las investigaciones realizadas respecto a la tecnología educativa y los medios de comunicación social se encaminan al qué y al cómo de su existencia y uso, que Martínez y Área (2003) asemejan a ferretería pedagógica, pero se adolece de trabajos que incluyan una dimensión axiológica. Sin embargo, Grill (1997) argumenta cómo lo primero que debe de hacer un profesional es buscar el porqué de las cosas desde planteamientos actitudinales, y señala que la tecnología no es un problema en sí mismo y sí el tecnopolismo entendido como los cambios éticos que pueden llegar a ocasionar en ámbitos laborales, por ejemplo, comportamientos adictivos a la tecnología o excesiva presión del uso de las mismas en dichos entornos.

Y es que la tecnología y los medios de comunicación social requieren de un análisis ético para utilizarlos de forma adecuada y coherente, como han resaltado Hawkrinde (1991), Nichols (1994), Postman (1995), o Sunstein (2003). Esto debe hacer su impronta en los procesos de orientación profesional, tanto los que se dan en los centros educativos, desde los niveles inferiores hasta los superiores, como en la familia u otros entornos exo y macrosistémicos (Bronfenbrenner, 1979), y bajo principios esenciales de la orientación (Repetto, Rus y Puig, 1994).

Este argumento se vincula con la línea de investigación Ciencia, Tecnología y Sociedad (CTS) surgida como oposición al modelo tecnológico unidireccional $(+$ ciencia $=+$ tecnología $=+$ riqueza $=+$ bienestar $)$, puesto que éste no respondía realmente a una verdadera concepción de la ciencia y tecnología, ya que en dicho proceso están inherentemente unidos elementos sociales como, entre otros, los valores morales, intereses profesionales, presiones políticas o condicionantes económicos, que otorgan una influencia notable en el origen y proceso científico-tecnológico (Bloor y Henry, 1996). En este sentido, López (2003) sugiere que, por un lado, se proporcione información humanística en forma de sensibilidad crítica a los estudiantes de ramas en ciencias naturales y científicas y, por otro lado, que se 
ofrezca un conocimiento acerca de la ciencia y tecnología a estudiantes de humanidades y ciencias sociales.

Al respecto, nuestra propuesta es doble e inclusiva: comprender los valores intrínsecos que conllevan las tecnologías (fin) y utilizar éstas como mediadoras para transmitir valores (medio). Desde aquí se expondrán diversos estudios en ambos sentidos, que se complementan con una experiencia investigadora que se ha desarrollado en dos centros de educación de adultos mediante el proyecto "Laboratorio de Observación sobre Tecnoética para Adultos" (LOTA). En la parte final del artículo se presentan algunas reflexiones referidas a la cuestión abordada.

\section{La orientación en tecnoética como fin y medio}

Como fin entendemos que las tecnologías y los mass-media incluyen en sí mismos una connotación valorativa. Con ello no se pretende utilizar un discurso únicamente negativo sobre dicho fin que, por otro lado, es el que habitualmente se ha realizado (Nichols, 1987; Ward, 2003; Medrano y Cortés, en prensa). La cuestión principal estriba en que las escuelas deben abordar estas cuestiones del ethos, como Katz (1992) las denomina, mediante el currículum o bajo una guía orientadora "to help us understand and diffuse the inevitable conflicts in our practice of educational technology". Tales conflictos pueden proceder de la influencia del mundo de las telecomunicaciones y para Dede (1986) es necesario que se atengan en la orientación educativa.

De igual manera, Braun (1992), Pruzan y Thyssen (1994), Postman (1995) y Bilbeny (1997) reflexionan sobre algunas acciones para trabajar en contextos escolares con el objetivo de analizar la revolución social que imprimen las computadoras en las competencias humanas. Pruzan y Thyssen (1994) proponen que en cada empresa se acuerde un código de valores, y que dicha idea se extrapole adaptándose a otros contextos, como el de la enseñanza, más en concreto la orientación profesional. Nos parece interesante su trabajo, así creemos que sería adecuada la figura de un mediador educativo, que podría ser el orientador, que plantease conflictos entre los valores que exige la sociedad tecnológica y de las telecomunicaciones y los que se inserten en el currículum para el desarrollo del alumno.

También, Bilbeny (1997) plantea una "revolución de los etemas", es decir, un mínimo común moral para el impacto tecnológico desde una perspectiva cognitivista y constructivista, que se basa en tres principios: pensar en uno mismo o principio de autonomía moral (moral point of view); imaginarse en el lugar del otro o principio de reciprocidad (ideal role-taking); y pensar de forma consecuente con uno mismo o principio de reflexibilidad (moral insight). Con ello, el autor se diferencia del discurso de Cortina (1998, 2001), que aboga por esa ética de mínimos aplicada a las TIC, como son: libertad, solidaridad, igualdad, responsabilidad y honestidad, que para Bilbeny serían máximos. En nuestra opinión, los valores que se deben seguir respetando son los que apunta Cortina, pero mediante los tres procedimientos que sugiere Bilbeny, es decir, la autonomía moral, la empatía, la reflexión, y aún sugerimos otro más, en cierta manera implícito: el compromiso sea cual sea nuestro rol social y profesional, aún más si se es educador (profesor, padre, monitor, orientador...). 
Bilbeny (1997) también sugiere aprender de nuevo de la sensibilidad, es decir, la recuperación de lo emocional, la empatía, la sensibilidad, la reflexión y la educación en las TIC. De esta forma, "nuestra comprensión de lo moral tiene que cambiar radicalmente con el crecimiento de la sociedad digital" (Bilbeny, 1997: 188). Otro aspecto que esgrime Bilbeny es el relacionado con lo espacial. Si con las tecnologías y los medios de comunicación existe un paso de una relación proximal, presencial y sensitiva, a una distal, virtual y subjetiva; este último vínculo tiene connotaciones relacionales y morales diferentes. En consecuencia una interacción distal puede invitar a que las personas conozcan otras diferentes, pero el peligro que Sunstein (2003) señala es que al final estos individuos a través de dichas formas virtuales acaban buscando espacios comunes, aislándose de otras experiencias e ideas. Sunstein (2003) en su libro Republica.com enfatiza que Internet es adecuado para fomentar un sistema democrático, porque aumenta los encuentros comunes entre sujetos, pero si al final estos acaban siendo muy selectivos y muy excluyentes, puede llegar a convertirse en un muro, es decir, una brecha digital o, en palabras de Castells (1997), una divisoria digital, que influye también en las diferencias profesionales.

Hasta el momento se ha analizado que la tecnoética como aspecto que conlleva una finalidad ética en sí misma debe tenerse en cuenta en la actual sociedad y en los ámbitos educativos a través de un compromiso y de propuestas de orientación educativa y profesional. Pero también, por otro lado, la orientación en tecnoética como medio significa que los propios medios tecnológicos y de información pueden ser transmisores y mediadores de contenidos y actividades de índole axiológica. Dicho planteamiento concuerda con algunas de las ideas de Ryan, Bednar y Sweeder (1999) sobre la necesidad de cultivar la moral mediante la tecnología educativa, por su vertiente motivadora con el fin de evitar el narcisismo, rasgo característico de la cultura americana según los autores, y una moralidad sustentada únicamente en el razonamiento. Los autores dan a conocer el proyecto denominado Social Projector Virual Gatherings, en el que entienden que la moralidad actualmente pasa por aunar los sentimientos y el comportamiento moral, y por ello, la simpatía, el deber, la imparcialidad o la justicia y el autocontrol. Con el proyecto existe la pretensión de que se logre que la justicia opere en las vidas de los educandos, así como que los sentimientos de deber y simpatía interaccionen en la práctica de la equidad personal y social. Y que ello se convierta en competencias (de saber ser) generales para cualquier ámbito profesional.

En concreto, para la práctica de la orientación educativa, Ryan et al. (1999) proponen cuatro tipos de estrategias: asambleas virtuales para trabajar temas éticos con la participación de diferentes personas usando el contacto virtual; la acción social mediante Internet como medio para buscar textos vinculados a temas de humanidad, trabajo comunitario o acciones solidarias; creación de simulaciones informáticas para abordar asuntos en los que hay que actuar y decidir como profesionales, por ejemplo, ante problemas de tipo medioambiental; y por último, producciones de vídeo para recrear historias "reales" que contengan un fondo ético a modo de role-playing.

También, el departamento de tecnología educativa del Estado de Utah ha creado un programa de aplicación tecnológica (Jensen, 1993) para estudiantes de grado medio y superior con el fin de orientar académica y profesionalmente en áreas como: tecnología industrial y agricultura; negocios y marketing; y, economía y ocupaciones de la salud. Presenta 18 se- 
siones, de índole práctica, con una duración media de 40 minutos como, por ejemplo, “¿Cómo soy yo?”, "Asesoramiento personal”, "Tomar una decisión”, "Decisión y emoción" e "Historias de casos reales sobre ocupaciones". Otro de estos módulos, que se acerca más a la temática que aquí se aborda, es "La escala de valores", en el que se ofrece información al estudiante sobre cómo se construye el desarrollo ético de una persona, aparece un cuestionario sobre qué valores sociales y éticos (placer, poder, reconocimiento, moralidad, creatividad, trabajo, etc.) son los más relevantes en su desarrollo profesional, se solicita que realice un orden de preferencia personal sobre sus valores $\mathrm{y}$, por último, aparecen unas preguntas de autoevaluación de la sesión.

Estas propuestas de incorporación de lo axiológico en los programas tecnológicos de orientación profesional son, en nuestra opinión, realmente interesantes, pero no lo es menos la necesidad de orientar en la formación de un profesional que ha de buscar el porqué de los hechos tecnológicos acerca de sus prácticas y actitudes (Grill, 1997). Se está de acuerdo con la idea de Repetto y Malik (1998) acerca de la importancia de aprender el uso de las TIC de forma afectiva, esto es, con el desarrollo de unas normas éticas y de calidad. Al respecto, la Association for Educational Communication and Technology (AECT) discute sobre la ética profesional relativa a investigaciones en entornos electrónicos colaborativos (Ravitz, 1997) mediante temas como la privacidad de datos de Internet y proliferación de información antisocial (grupos racistas, pornografía infantil...). Ambas problemáticas, de rabiosa actualidad, quizá podrían ser un tema de análisis educativo en ramas técnicas y humanísticas en la línea apuntada más arriba (López, 2003).

La orientación dentro de la sociedad del conocimiento ha de considerarse en dos sentidos: alfabetización en las TIC y "alfabetización" en la no discriminación e igualdad de acceso a éstas. En este último sentido son cada vez más las voces (Pantoja, 2004; Touriñan, 2004; Ortega, 2004) que sugieren que aprender a aprender y aprender a vivir juntos han de introducirse como contenidos en el aprendizaje de las TIC para formar ciudadanos que buscan información sobre los hechos bajo una pluralidad y democratización en dicha información.

Los estudiantes han de encaminarse a la alfabetización tecnológica, pero acompañada de otro tipo de aprendizajes más humanos y sociales (Flecha y Rotger, 2004). Algunos autores identifican esto último con responsabilidad o deontología profesional. Como expresa Martínez (2003), la educación de un futuro profesional no sólo debe ser la de resolver problemas, sino que debe adquirir una ocupación moral puesto que muchas de las decisiones en la práctica laboral conllevan un conflicto de valores. Por ejemplo, un conflicto de intereses entre estándares como es la de un trabajador en guardar confidencialidad de la información laboral o su divulgación y/o denuncia. Por su lado Pantoja (2004) menciona el dilema entre la modernización de los sistemas profesionales y la no discriminación e igualdad de acceso a la formación. También se puede añadir el aislamiento de las personas frente al fomento de las relaciones interpersonales (como ocurre en el teletrabajo o los trabajos en red).

En resumen, se defiende la inclusión de la tecnología como objetivo y contenido de la orientación profesional desde tres ángulos (ver gráfica 2): saber sobre las tecnología, saber cómo utilizarlas y saber analizarlas críticamente, aspecto último en el que hemos basado nuestro discurso anterior. 


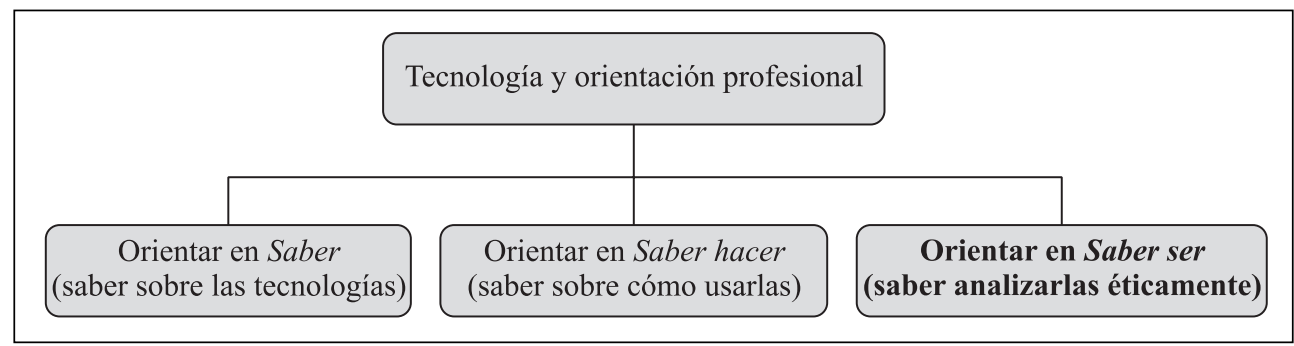

\section{GRÁFICA 2.}

Relación entre saber, saber hacer y saber ser en la tecnología y orientación profesional.

\section{Una aportación investigadora: Laboratorio de Observación de Tecnoética para Adultos (LOTA)}

Toda esta contextualización epistemológica es parte de la génesis del proyecto "Laboratorio de Observación sobre Tecnoética para Adultos" (LOTA) ${ }^{1}$, que se ha desarrollado en dos centros de educación permanente, bajo la coordinación de la autora de este artículo, durante 2003-2005. La mayoría de las propuestas en el terreno de la educación de adultos han ido encaminadas a la formación a distancia, las tecnologías como prótesis de la memoria o Internet para la relación intergeneracional y generacional. Sin embargo en los programas de orientación educativa y profesional dirigidos a los adultos se carece de ese planteamiento que se está defendiendo, más reflexivo sobre los recursos y medios de comunicación de repercusión social. Como contribución a una solución posible, se ha iniciado el proyecto LOTA, cuyo fin es enseñar a saber analizar, criticar, elegir y reflexionar sobre las nuevas tecnologías de la información y la comunicación a través de una interpretación axiológica.

El proyecto ha contado durante 2003-2004 con el desarrollo de una serie de módulos: "Recogida de conocimientos previos y presentación del proyecto" (Cortés, 2005b), "Análisis crítico y valorativo de los instrumentos mediáticos televisivos y de radio", "Debate sobre lo positivo y negativo de las tecnologías", "Dilemas relacionados con las TIC", "Dramatización sobre las TIC", "La influencia de la publicidad en el éxito profesional", e "Internet en el mundo laboral". En el curso 2004-2005 se creó un soporte multimedia: página web (http://usuarios.lycos.es/tecnoeticazgz), CD y material impreso con toda la información y resultados del proyecto, además de organizar unas jornadas sobre la "Credibilidad de los medios de comunicación", es decir, sobre el código ético de los profesionales de la comunicación y la información. En un futuro, nos gustaría hacerlo extensible a más profesiones. A continuación se presentan conclusiones muy generales de algunos de estos módulos. De antemano, quisiéramos señalar que esta propuesta de LOTA ha sido evaluada con un 4,2 de media en una escala del 1 (muy negativamente) al 5 (muy positivamente) por un total del $72 \%$ de los adultos encuestados al final del proyecto.

\footnotetext{
Proyecto aprobado por la Diputación General de Aragón en las convocatorias de 2003 y 2004, dirigido por Carlos Sanz, director del centro Concepción Arenal (Zaragoza) y coordinado por la firmante de este trabajo. También colabora Isabel Segura (Teruel).
} 
En el primer módulo indagábamos las preconcepciones de los adultos (Cortés, 2005b), que fueron 150 (110 mujeres y 40 hombres) entre 18 y 63 años. Así una pregunta del cuestionario que respondían era la siguiente: ¿Crees que Internet ha cambiado el mundo laboral y la sociedad? ¿En qué? Ante dicha pregunta el análisis, siguiendo a Pascual-Leone (1978), ha sido el que aparece en la tabla 1.

TABLA 1: Tabla de contingencia de los conocimientos previos sobre Internet.

\begin{tabular}{|l|c|c|c|c|}
\hline & Frecuencia & Porcentaje & Porcentaje válido & Porcentaje acumulado \\
\hline Nada & 50 & 32,9 & 33,3 & 33,3 \\
\hline Anecdótico & 62 & 40,8 & 41,3 & 74,7 \\
\hline Impreciso & 36 & 23,7 & 24,0 & 98,7 \\
\hline Elaborado & 2 & 1,3 & 1,3 & 100,0 \\
\hline Total & 150 & 98,7 & 100,0 & \\
\hline
\end{tabular}

Es decir, se escinden las preconcepciones en tres tipos, de mayor a menor cercanía con la definición más consensuada según el concepto de Internet (Castells, 1997), esto es, elaborado o globalizado (el más idóneo); impreciso o puntual (el que describe una idea idónea, pero no de forma total, sino aportando una concepción parcial); y anecdótico u ocasional (el que describe el concepto, con sólo algún dato idóneo y/o con algún ejemplo).

Así, las respuestas se acercan a una definición anecdótica con la idea de que "une, pero crea adicción y por eso separa a la gente" $(40,8 \%)$, con protocolos como que dicha red facilita conocer gente fácilmente, ayuda a la comunicación, crea adicción y separa a las personas. En segundo lugar, 50 sujetos no ofrecen respuesta alguna, y en tercer lugar sí lo hacen 36 adultos con contenidos cercanos al nivel impreciso, sólo uno en el elaborado. Un hombre de 53 años comenta lo siguiente "Internet une en la distancia y separa en la proximidad. Ayuda a conocer lo que está lejos y buscamos lejos lo que ignoramos cerca". De aquí, podríamos inferir que la reflexión axiológica de los sujetos del trabajo respecto a la influencia de Internet es más bien escasa, es decir, anecdótica. En nuestra opinión, este análisis o diagnóstico debería tenerse en propuestas de orientación e intervención profesional, puesto que Internet es un medio de trabajo casi imprescindible que cada vez se conoce y se utiliza más y que por ende, dados estos datos, tendría que potenciarse un análisis más crítico y axiológico sobre todas sus repercusiones laborales y personales.

Otro ejemplo lo encontramos en el módulo tercero, esto es, un debate que se propondría en clase sería el siguiente: "Supongamos que somos miembros de un comité de evaluación que determina si debe o no dar fondos económicos a un proyecto de investigación sobre la fecundación in vitro. ¿Tendríamos que considerar los aspectos éticos o basarnos únicamente en principios científicos? ¿Por qué sí, o por qué no? Y en su caso, ¿cuáles serían tales principios éticos?". En esta actividad participaron 79 alumnos, 46 mujeres y 33 hombres, entre 18 y 56 años. La mayoría, un 85\%, se situaron como partidarios de considerar dichos aspectos éticos, pero hubo discrepancias en cuanto a si esa ayuda económica tenía que ser pública o no; ya que si partía de fondos públicos, un primer principio era que los resultados fuesen beneficiosos para toda la población. Otro principio con el que hubo un relativo 
acuerdo $(65 \%)$ es que no perjudicase vitalmente a ningún humano. Se apreció favorablemente $(70 \%)$ el que los científicos en sus diferentes carreras universitarias cursasen algunas materias relacionadas con la ética en las ciencias. Un $75 \%$ de los adultos evaluaron con un 5 (muy positivamente), en una escala del 1 al 5, dicho debate, siendo éste el módulo mejor valorado.

En el módulo sobre "Dilemas relacionados con las TIC" se les pasó, en forma de entrevista, un dilema hipotético y posteriormente se solicitó uno real, que contestaron 82 sujetos (52 mujeres y 30 hombres). Los dilemas reales se han clasificado, con un análisis interjueces entre 3 personas, en cinco categorías:

1) Credibilidad de los MCS (dilemas en los que se contraponen los valores de objetividad frente a la subjetividad o manipulación sensacionalista desde los mass media), con un $37 \%$;

2) Calidad de vida en el trabajo(valores contrapuestos sobre si con las TIC se gana o se pierde tiempo en la realización de tareas laborales), con un $27 \%$;

3) Salud (utilizar las TIC o no porque la repercusión de las ondas tecnológicas en el ser humano), con un $18 \%$;

4) Discriminación social (se plantean si las TIC son sinónimo de poder o pueden fomentar la igualdad porque dan ayuda a todos, es decir, son para toda la humanidad o si sólo para el enriquecimiento de unos privilegiados), con un $10 \% \mathrm{y}$;

5) Utilidad de Internet (si poner Internet en casa o en el trabajo o no por dudas sobre su eficacia, rapidez, facilidad, etc.), con un $8 \%$.

Para finalizar, en el último módulo, tres profesionales del mundo de la radio, la televisión y el periodismo, aportaron su visión respecto a cómo los mass-media ejercen su función de credibilidad. Sólo señalamos dos conclusiones: la objetividad en los medios de comunicación es imposible, y depende estrechamente de la ideología del grupo editorial o de comunicación; pero debe haber unos límites deontológicos acordados entre todos y en los que participen voces del Gobierno, los telespectadores, oyentes y/o lectores. Asimismo, se discutió y se llegó a un consenso sobre que los principios, ideados entre el Gobierno y las televisiones públicas y privadas, en el Acuerdo para el fomento de la Autorregulación sobre contenidos televisivos e infancia $(2005)^{2}$ han de ser incluidos en la orientación y formación de un futuro profesional, en este caso, de los medios de comunicación social; en concreto, el de la televisión.

\section{Algunas reflexiones: hacia un análisis axiológico de la orientación profesional}

Llegados al final y a partir de lo expuesto, queremos concluir con algunas ideas que sería necesario se atendiesen en un futuro en los campos educativos formales. Las iniciativas e-learning y e-orientación requieren de esta perspectiva (orientar en saber ser con las $\mathrm{TIC}$ ) en la formación para el empleo, puesto que éste evoluciona en consonancia con las características socioeconómicas y los avances tecnológicos. La orientación debe estar alerta a estos movimientos no sólo para crear profesionales eficaces técnicamente, sino también

2 Ver http://www.cnice.mecd.es/tv_mav/n/f6_normativa.htm. 
ciudadanos responsables y que sepan vivir juntos. Quizá, de esta forma, dichas iniciativas europeas mejoren en su funcionamiento como argumenta Pantoja (2004).

Por otra parte, se defiende que la relación entre la responsabilidad oficial y la responsabilidad profesional es crucial para entender muchos aspectos y limitaciones de esta última, y asimismo es esencial el vínculo con el deber educativo. Se ha presentado una iniciativa real en educación de adultos (LOTA), que ha tenido un resultado muy satisfactorio por parte de los receptores del mismo, como se ha descrito. También los profesores participantes valoraron positivamente esta propuesta. Quizá, la consecuencia más reseñable es que el curso 2005-2006, a pesar de haber finalizado el proyecto, algunos profesores han realizado actividades en la línea de los módulos implementados en el proyecto LOTA.

Pero, en este punto también se sugieren otras propuestas en distintas etapas en la educación, esto es, primaria, secundaria, y postsecundaria, para una orientación en tecnoética a lo largo del ciclo vital. Así, en la enseñanza primaria y secundaria, desde nuestra perspectiva, proponemos la inserción en el currículum de forma transversal a las asignaturas estos temas a través de actividades como, por ejemplo, "Internet: brecha y puente" para analizar la frontera digital de la red entre los que acceden y no a todo el mundo de la red, así como la posibilidad de crear lazos y de difundir información, y sus repercusiones laborales de inclusión y exclusión, o "El ordenador" para tratar las potencialidades y limitaciones del ordenador (juegos de ordenador, programas informáticos, ...) en las relaciones sociales, orientación académica e inserción laboral. Creemos que un modo acertado de abordar estos temas es a través del uso de estrategias de intervención en valores como, entre otras, dilemas éticos, trabajo por grupos, técnicas de clarificación de valores, debates o role-playing. Además de valernos de recursos materiales tanto impresos, como tecnológicos y audiovisuales (vídeos, diapositivas, foros educativos, Internet, ...) (Cortés, 2004).

Cada profesión posee un código deontológico sobre qué normas y principios rigen su práctica. Por lo tanto, en las ramas o módulos de formación profesional y campos universitarios tiene que estudiarse este contenido al igual que otras cuestiones más técnicas, conceptuales y procedimentales. Ello, en parte, se intenta recoger en el nuevo Espacio Europeo de Educación Superior, en el hecho de que los alumnos no sólo aprendan contenidos, sino actitudes a través de las competencias participativas y personales. Y sería necesario analizarlo sea cual sea el itinerario de formación y ámbito ocupacional, pero en nuestro caso nos referimos más específicamente a aquéllos que manipulen instrumentos tecnológicos y estén inmersos en las telecomunicaciones, por ejemplo un ingeniero, un científico, un administrativo, un programador de ordenadores o un director de un informativo televisivo. Aunque, también y por supuesto, en la formación de un futuro educador y orientador, que requiere conocerlo para luego saber asesorar en dicha perspectiva. Una experiencia (Cortés, 2004), en este sentido, se realizó, con resultados positivos, mediante un seminario aplicado en la materia de Nuevas Tecnologías en Magisterio, con el fin de que cuando los alumnos fuesen maestros supiesen incluir actividades tecnológicas con un análisis ético.

En esta dirección, resulta apreciable que actualmente con la impronta de todo el mundo de la tecnología y la comunicación, y de las ciencias de la vida, están surgiendo muchos debates en torno a los códigos deontológicos. Camps (2003) apunta a que cada vez son más necesarios los comités de expertos para discutir y, eventualmente, adoptar decisiones sobre aspectos éticos relacionados con la protección, ante todo, de la persona y del medio ambiente. De todas maneras estos temas no sólo pueden tratarse en la formación inicial, sino 
que también atraviesan la formación continua como es el caso de la educación con adultos, a la que se encamina el proyecto aquí señalado, el LOTA. En dicho proyecto se ha intentado que a través de diferentes módulos los alumnos fuesen acercándose a diferentes reflexiones sobre las repercusiones éticas que albergan el uso de las TIC y los medios de comunicación en su vida diaria y laboral. Habría que reseñar que el LOTA ha sido favorablemente valorado por los alumnos.

Seguimos a Hargreaves (1999) cuando reclama que una vía de estudio esencial en la actualidad es el desarrollo de las competencias de la gente en las nuevas tecnologías de una manera crítica y reflexiva. Consecuentemente, se aboga y se invita a investigar en la línea aquí propuesta, esto es, orientar profesionalmente en saber ser con las TIC.

\section{Referencias bibliográficas}

Acuerdo para el fomento de la Autorregulación sobre contenidos televisivos e infancia. (2005). Gobierno de España y Televisiones españolas. http://www.cnice.mecd.es/tv_mav/n/f6_normativa.htm Consultado en abril de 2005.

Asociación Internacional de Orientación Educativa y Psicopedagógica (AIOEP, 2003). Competencias Internacionales para los Profesionales de Orientación y Educación. Consultado el 14 de abril de 2004 en http://www.iaevg.org/iaevg/nav.cfm?lang=4\&menu=1\&submenu=5.

Bilbeny, N. (1997). La revolución en la ética. Hábitos y creencias en la sociedad digital. Barcelona: Anagrama.

Bloor, D. y Henry, J. (1996). Scientific Knowledge: A Sociological Analysis. Londrés: Athlone.

Braun, J. (1992). "Caring, citizensship, and conscience. The cornestones of a values education curriculum for elementary schools". International jorunal of social education, 7 (2), 47-56.

Bronfenbrenner, U. (1979). The ecology of human development: Experiment by nature and design. Cambridge: Harvard University Press.

Cabero, J. (2001). Tecnología educativa. Diseño y utilización de medios en la enseñanza. Barcelona: Paidós.

Camps, V. (2003). "Ética para las ciencias y técnicas de la vida". En A. Ibarra y L. Olivé (Eds.), Cuestiones éticas en ciencia y tecnología en el siglo XXI (pp. 225-244). Madrid: Biblioteca Nueva.

Castells, M. (1997). La era de la información. Economía, sociedad y cultura. (3 vols). Madrid: Alianza.

Cogoi, C. et al. (2005). "Ict Skills For Guidance Practitioners: Final Results Of The Research. Grupo de Trabajo, CD-rom" en AIOSP Conferencia Internacional. Lisboa.

Cortés, P. A. (2004). Una mirada psicoeducativa de los valores. Seminario aplicado a las nuevas tecnologías de la educación. Prensas Universitarias de la Universidad de Zaragoza: Zaragoza.

Cortés, P. A. (2005a). "Educational Technology: as a means to an end". Educational Technology Review, 13 (1), 73-90.

Cortés, P. A. (2005b). "Las preconcepciones sobre la tecnoética en los adultos". Revista Mexicana de Psicología, 22, 2, 541-552.

Cortés, P. A. (2006). "Valores y orientación profesional: líneas de investigación e intervención actuales". Contextos Educativos, 8-9, 223-238.

Cortina, A. (1998). “¿Qué son los valores y para qué sirven?”. Temas para el debate, 42, 20-22.

Cortina, A. (2001). Alianza y Contrato. Política, ética y religión. Madrid: Editorial Trotta. 
Dede, C. (1986). "The implications of emerging technologies for the value-oriented curriculum". Momentum, 61, 42-44.

Flecha, R. y Rotger, J. M. (2004). "Innovación, democratización y mejora de la docencia universitaria en el marco de la Sociedad de la Información". Contextos Educativos, 6-7, 159-166.

Gill, D. W. (1997). "Educating for meaning and morality: The contribution of technology". Bulletin of Science, Technology and Society, 17, 5-6, 249-260.

Hargreaves, A. (1999). Profesorado, cultura y postmodernidad. Madrid: Ediciones Morata.

Hawkridge, D. (1991). "Challenging educational technology". ETTI, 28, 2, 102-110.

Katz, S. B. (1992). "The ethic of expediency: Classical Rhetoric, Tecnology, and the Holocaust". College English, 54, 3, 255-275.

López, J. A. (2003). "Ciencia, técnica y sociedad”. In A. Ibarra \& L. Olivé (Eds.), Cuestiones éticas en ciencia y tecnología en el siglo XXI (pp. 113-158). Madrid: Biblioteca Nueva.

Jensen, R. (1993). TLC Career Guidance Currículo. Utah: Salt Lake City.

Martínez, S. (2003). "Ética de científicos y tecnólogos". In A. Ibarra \& L. Olivé (Eds.), Cuestiones éticas en ciencia y tecnología en el siglo XXI (pp. 277-300). Madrid: Biblioteca Nueva.

Martínez, F. y Área, M. (2003). El ámbito docente e investigador de la tecnología educativa en España. Algunos apuntes para el debate. Paper presented at the meeting of the Reunión del área de Didáctica y Organización Escolar, Valencia, Universidad de Valencia.

Medrano, C. y Cortés, P. A. (en prensa). "Teaching and learning of values through television”. International Review of Education.

Medrano, C. et al. (en prensa). "La televisión y el desarrollo de valores”. Revista de Educación.

Nichols, R. G. (1987). "Toward a conscience: Negative aspect of educational technology". Journal of visual/Verbal Languaging, 7 (1), 121-137.

Nichols, R. G. (1994). "Searching for moral guidance about educational tecnology". Educational technology, 34 (2), 40-48.

Ortega Ruiz, P. (2004). "La educación moral como pedagogía de la alteridad". Revista Española de Pedagogía, 227, 5-30.

Pascual-Leone, J. (1978). "La teoría de los operadores constructivos". En J. Delval (Comp.). Lecturas de psicología del niño (pp. 25-36). Madrid: Alianza.

Postman, N. (1993). Technopoly: The Surrender of Cultura to technology. New York: Vintage.

Pantoja, A. (2004). La intervención psicopedagógica en la sociedad de la información. Educar y orientar con nuevas tecnologías. Madrid: Editorial EOS.

Postman, N. (1995). "Making a living. Making a Life: Technology reconsidered". College Board Review, 176-177, 8-13.

Pruzan, P y Thyssen, O. (1994). "The renaissance of ethics and the ethical accounting statement". Educational Technology, 34 (1), 23-28.

Ravitz, J. (1997). "Ethics in scholarly communications: intellectual property and new technologies". In National Convention of the Association for Educational Communications and Technology, $19^{\text {th }}$, Albuquerque.

Repetto, E., Rus, V. y Puig, J. (1994). Orientación educativa e intervención psicopedagógica. Madrid: UNED.

Repetto, E. y Malik, B. (1998). "Nuevas tecnologías aplicadas a la orientación”. En R., Bisquerra, Modelos de orientación e intervención psicopedagógica. Barcelona: Praxis. 
Ryan. J. et al. (1999). "Tecnology, narcissism, and the moral sense: implication for instruction". British Journal of Educational Technology, 30, 115-128. 1999.

Sunstein, C. R. (2003). República.com. Internet, democracia y libertad. Barcelona: Paidós.

Touriñan, J. M. (2004). "La educación electrónica: un reto de la sociedad digital en la escuela“. Revista Española de Pedagogía, 227, 5-30.

Ward, L. M. (2003). "Understanding the role of the entertainment media in the sexual socialization of American youth: A review of empirical research". Developmental Review, 23, 347-388.

Fecha de recepción: 01-02-05

Fecha de revisión: 06-03-06

Fecha de aceptación: 17-10-06 